The authors analysed the metabolic potential of the dominant organisms in the samples by annotating the probable functions of the genes identified and noting putative metabolic pathways in the assembled draft genomes. Three particularly interesting types of gene were widespread. These encode enzymes that aid the oxidation of trace gases in energy-generating cellular pathways. The enzymes are: highaffinity hydrogenases, CoxLSMs (high-affinity carbon monoxide dehydrogenases) and type IE RuBisCOs ( $\mathrm{CO}_{2}$-fixation enzymes).

The proposed role of these key genes in tracegas oxidation was supported by biochemical analyses, in which the authors demonstrated that the same soil samples showed substantial and reproducible uptake of hydrogen and carbon monoxide, associated with increased $\mathrm{CO}_{2}$ fixation. This fixation process was not increased by light, indicating that photosynthetic processes were not involved.

However, caution might be called for when interpreting these findings. The genomicanalysis approach used by the authors can be biased towards organisms present in high abundance ${ }^{5}$. Even if cyanobacteria occur in low abundance, they might still make substantial contributions to key ecosystem services. The authors note that both photosynthesis and the trace-gas metabolism that they describe could co-occur in Antarctic soils, with the dominant process probably dependent on physicochemical factors such as water availability.

Ji et al. are careful to note that their evidence for this bioenergetics mechanism is restricted to some localized and specialized extreme Antarctic habitats. However, they propose that trace gases might be a more widespread energy source, having identified the key genes required for this metabolic process in several publicly available microbial DNA-sequence data sets obtained from sampling of an aridsoil region of Antarctica called the McMurdo Dry Valleys (Fig. 1). Yet why has this phenomenon not been observed previously in Antarctic soils? The probable reason is that no organisms showing this particular physiology have been cultured from Antarctic soils, and in desert soils in less-extreme environments, the dominant energy-acquisition process is cyanobacterial photosynthesis ${ }^{6}$.

The generation of energy through hydrogen and carbon monoxide metabolism has been observed before ${ }^{7}$. A wide range of microbes use hydrogen as a sole energy source to support $\mathrm{CO}_{2}$ fixation, although mostly under anaerobic (oxygen-free) conditions. Bacteria such as Geobacter sulfurreducens ${ }^{8}$ can scavenge atmospheric carbon monoxide as their sole energy source ${ }^{9}$, and carbon monoxide dehydrogenase genes are widespread in other strictly anaerobic extreme environments, such as deep sub-sea-floor sediments ${ }^{10}$.

There might be scepticism that the aerobic energy-capture process proposed by Ji and colleagues could suffice to maintain a microbial community. It seems instinctively implausible that the hydrogen and carbon monoxide levels are high enough in the cold, clear atmosphere of Antarctica to support life. Although the authors do not report the local atmospheric gas concentrations of the Robinson Ridge or Adams Flat sampling sites, they do present calculations showing that the oxidation of hydrogen and carbon monoxide can theoretically support the energy requirements of microbial populations in the Antarctic soil.

It is worth remembering that life in these environments is not exactly fast-paced, and cells are inactive for much of any year. The entire annual metabolic window - the period in which the organisms can be metabolically active - might be only a few hundred hours per year (compared to many thousands of hours for a moist soil in a temperate climate). Perhaps cell doubling takes days or months, rather than the minutes or hours needed in less-extreme environments.

Definitive proof of this proposed physiology will require the eventual isolation and laboratory culturing of these organisms, and the in vivo demonstration that such microbes can grow using hydrogen and carbon monoxide as energy sources. What is particularly exciting about Ji and colleagues' findings, especially for astrobiologists, is that it adds another dimension to our understanding of the survival and energy-sufficiency mechanisms of organisms living in places at the limits of where life is thought to be possible. The results might also stimulate further studies into the extent of hydrogen metabolism in other extreme environments.

Don A. Cowan and Thulani P. Makhalanyane are in the Department of Genetics, Centre for Microbial Ecology and Genomics,

University of Pretoria, Hatfield 0028, Pretoria, South Africa.

e-mail:don.cowan@up.ac.za

1. Bockheim, J. G. in Antarctic Terrestrial Microbiology (ed. Cowan, D. A.) Ch. 16 (Springer, 2014).

2. Cary, S. C., McDonald, I., Barrett, J. E. \& Cowan, D. A. Nature Microbiol. Rev. 8, 129-138 (2010).

3. Ji, M. et al. Nature 552, 400-403 (2017).

4. Chan, Y., Van Nostrand, J. D., Zhou, J., Pointing, S. B. \& Farrell, R. L. Proc. Natl Acad. Sci. USA 110 8990-8995 (2013).

5. Wu, Y.-W., Tang, Y.-H., Tringe, S. G., Simmons, B. A. \& Singer, S. W. Microbiome 2, 26 (2014).

6. Wood, S. A., Rueckert, A., Cowan, D. A. \& Cary, S. C. ISME J. 2, 308-320 (2008).

7. Conrad, R. Microbiol. Rev. 60, 609-640 (1996).

8. Geelhoed, J. S., Henstra, A. M. \& Stams, A. J. M. Appl. Microbiol. Biotechnol. 100, 997-1007 (2016).

9. Lalonde, I. \& Constant, P. Appl. Environ. Microbiol. 82, 1324-1333 (2016)

10. Hoshino, T. \& Inagaki, F. Lett. Appl. Microbiol. 64, 355-363 (2017).

This article was published online on 6 December 2017.

\title{
IMMUNOLOGY
}

\section{The origins of memory T cells}

\begin{abstract}
Memory T cells protect against previously encountered pathogens, but their origins are unclear. Two studies track DNA modifications over time and find that these cells arise from effector T cells. SEE ARTICLE P.362 \& LETTER P.404
\end{abstract}

\section{KYLA D. OMILUSIK \& ANANDA W. GOLDRATH}

V accination has substantially reduced illness and death from infectious disease by exploiting the ability of long-lived memory T cells to 'remember' a previous encounter with a specific microbe and mount a rapid response upon pathogen re-exposure. Understanding how immunological memory is established and maintained might provide insights that could enable improvements in vaccine design. In this issue, Akondy et al. ${ }^{1}$ (page 362) and Youngblood et al. ${ }^{2}$ (page 404) reveal the cell population that gives rise to memory $\mathrm{T}$ cells, and how the population of memory T cells evolves.

Naive T cells are those that haven't previously responded to a pathogen. When they recognize a pathogen, they rapidly divide and express molecules such as cytokine proteins that help to fight infection. These responding cells are called effector T cells (more specifically, a type of effector cell called a cytotoxic $\mathrm{T}$ cell) and they can migrate into inflamed tissues and kill infected cells ${ }^{3}$. Once the pathogen is eliminated, most effector cells die, but a small pool of long-lived memory cells remains that is poised to respond rapidly if reinfection occurs $^{3}$.

Which cells give rise to memory $\mathrm{T}$ cells has been extensively investigated. Two general possibilities (Fig. 1a, b) have been proposed: the cells either arise from a subset of the effector cells that escape death, or instead descend directly from naive T cells, which could, as early as their first cell division, give rise to cells with effector-T-cell or memory-T-cell potential $^{3}$. 


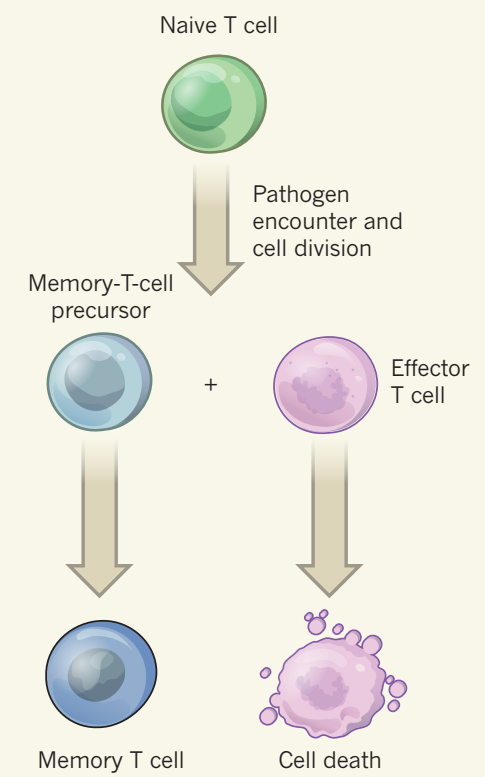

b

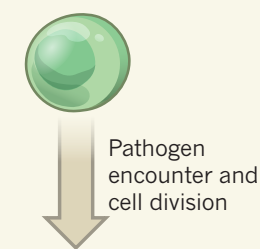

c

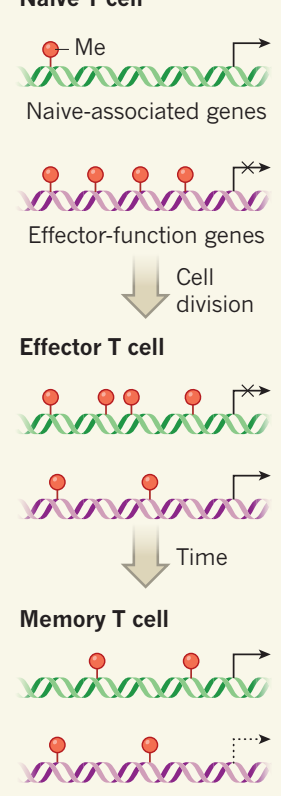

Figure 1 | The formation of memory $\mathrm{T}$ cells. When naive $\mathrm{T}$ cells encounter a pathogen, they proliferate, giving rise to many effector $\mathrm{T}$ cells that fight infection and die when the infection is over. Pathogen infection also results in the formation of long-lived memory $\mathrm{T}$ cells that rapidly respond to a previously encountered pathogen. a, One model for memory-T-cell formation proposes that the precursor cells that give rise to memory $\mathrm{T}$ cells and effector $\mathrm{T}$ cells both arise independently from naive $\mathrm{T}$ cells. $\mathbf{b}$, In another model, a subset of effector cells gives rise to memory T cells. c, Akondy et al. ${ }^{1}$ and Youngblood et al. ${ }^{2}$ studied memory-T-cell formation in human T cells and in vivo in mice, respectively. The authors monitored the addition of methyl $(\mathrm{Me})$ groups to DNA, a change usually associated with a decrease in gene expression. They analysed the methylation of genes associated with naive-cell function and effectorcell function in naive cells, effector cells and memory cells to reveal the cells' developmental histories. The authors also analysed the patterns of DNA accessibility (not shown) in the different cell types. They find that effector-function genes have similar methylation patterns in effector $\mathrm{T}$ cells and memory $\mathrm{T}$ cells, which could allow memory $T$ cells to rapidly re-express (dotted arrow) those genes upon pathogen reinfection. Their evidence is consistent with the model shown in $\mathbf{b}$.

The two new studies aimed to resolve this debate by tracking $\mathrm{CD} 8^{+} \mathrm{T}$ cells (those that express the protein CD8 on their cell surface) during the course of an infection. Akondy et al. ${ }^{1}$ studied people who received a vaccine for yellow fever virus, whereas Youngblood et al. ${ }^{2}$ worked with a mouse model of lymphocytic choriomeningitis viral infection. Both studies examined populations of naive, effector and memory $\mathrm{T}$ cells for epigenetic modifications - heritable chemical modifications or structural changes to a cell's DNA that do not alter the DNA sequence. Such changes are often associated with the regulation of gene expression, allowing a cell and its descendants to 'bookmark' a particular geneexpression pattern. DNA methylation is one such alteration that can fix genes in an 'off' position, thus silencing their expression ${ }^{4}$.

Akondy and colleagues also examined the DNA configuration in T cells to identify genomic regions tightly packaged in a 'closed' state, which are not accessible to the geneexpression machinery, and regions in an 'open' state that allows gene expression ${ }^{5}$. Although RNA profiling can provide a snapshot of the genes currently being transcribed by a cell, the approaches taken by the authors to analyse epigenetic changes provide insight into the path by which a given transcriptional state is reached.

Using genome-wide DNA-methylation profiling, Youngblood and colleagues found that, as naive $T$ cells differentiated into effector cells, their DNA-methylation profile changed. Methyl groups were added to many genes associated with the naive state, whereas a loss of DNA methylation was observed at genes that encode key components of the effector response.

The authors identified the DNA methyltransferase Dnmt3a as a key enzyme responsible for de novo DNA methylation during the immune response. They also showed that, in effector $\mathrm{T}$ cells that are differentiating into memory cells, the methylation of genes expressed in the naive state can be removed in a cell-division-independent process that might drive the re-expression of naive-associated genes, which could, in turn, be needed to establish or maintain the long-lived memorycell population.
Importantly, both studies conclude that, although memory $\mathrm{T}$ cells no longer express effector molecules, the genes that encode these molecules remain in a state of low methylation (Fig. 1c). Akondy and colleagues also reveal that memory $\mathrm{T}$ cells that are present as long as ten years after vaccination have an open genome-packaging pattern that is comparable to that found in effector T cells, even though these memory $\mathrm{T}$ cells do not divide or express effector molecules until reactivated by pathogen encounter. Thus, effector-associated genes were maintained in memory $\mathrm{T}$ cells in a configuration that more closely resembled that of effector T cells than that of naive-T-cell populations. This is consistent with the ability of memory cells to re-express effector molecules quickly to fight reinfection ${ }^{3}$.

Both studies provide strong evidence for a model in which memory $\mathrm{T}$ cells descend from a population of cells that have previously expressed genes associated with effector-T-cell function, and have turned off effector-gene expression but retained a 'memory' of their developmental path. This takes the form of DNA modifications that would enable them to rapidly become an effector cell upon pathogen reinfection. In essence, epigenetic modifications bookmark the chapter of infection in a memory T cell's history, allowing the cell to rapidly 'recall' its effector capacity.

Although inferring T-cell lineages by isolating bulk populations and comparing heritable epigenetic states is illuminating, it does not exclude the possibility that a small population of naive T cells could also independently form memory cells directly without going through an effector-T-cell phase. Akondy and colleagues addressed this possibility by directly labelling dividing effector T cells in individuals using a heavy form of hydrogen called deuterium.

Virus-specific memory $\mathrm{T}$ cells that were present between one and two years after vaccination showed little dilution of the deuterium, indicating that minimal cell division had occurred in these cells. In a comparison of cell-surface proteins, these memory cells resembled naive $\mathrm{T}$ cells, but the deuterium labelling reveals that they were formed from the dividing effector-T-cell population. These results support a model in which viral-specific $\mathrm{CD} 8^{+} \mathrm{T}$ cells extensively proliferate upon pathogen recognition and modify their DNA to favour expression of effector molecules. Later the cells stop dividing, stop expressing effector genes and reexpress many genes associated with the naive state, such as genes that aid T-cell survival and migration.

Not all memory T cells are the same: a proportion of them, like naive cells, circulate throughout the body waiting to be called back into action, whereas tissue-resident memory cells reside in tissues such as the lung, skin and gut, providing the first line of defence against 
reinfection at sites where pathogens will often first enter the body ${ }^{6}$. Whether these different types of memory $\mathrm{T}$ cell arise through the same pathway remains to be determined.

These two studies suggest that a goal of vaccine design should be to stimulate a large, robust response from the effector $\mathrm{T}$ cells from which memory T-cell populations can arise. However, the conditions that best promote effector cells to become memory cells still need to be fully defined. Therapeutic targeting of the DNA-modification machinery, such as Dnmt3a, might prove to be a useful strategy to increase vaccine efficacy.

Kyla D. Omilusik and Ananda W. Goldrath are in the Division of Biological Sciences, University of California, San Diego, La Jolla, California 92093, USA.

e-mail:agoldrath@ucsd.edu
1. Akondy, R. S. et al. Nature 552, 362-367 (2017).

2. Youngblood, B. et al. Nature 552, 404-409 (2017).

3. Kaech, S. M. \& Cui, W. Nature Rev. Immunol. 12 , 749-761 (2012).

4. Winter, D. R., Jung, S. \& Amit, I. Nature Rev. Immunol. 15, 585-594 (2015)

5. Gray, S. M., Kaech, S. M. \& Staron, M. M. Immunol. Rev. 261, 157-168 (2014)

6. Chang, J. T., Wherry, E. J. \& Goldrath, A. W. Nature Immunol. 15, 1104-1115 (2014).

This article was published online on 13 December 2017.

\section{Martian water stored underground}

\section{Why did Mars lose so much of its surface water, whereas Earth retained its? Models of the evolution of minerals on the two planets suggest one explanation: the Martian water was drawn into the planetary interior. SEE LETTER P.391}

TOMOHIRO USUI

$\longrightarrow$ bservations of Mars and its geology suggest that the red planet once had an Earth-like hydrological cycle that included large lakes or oceans. In contrast to this ancient wet environment, the surface of Mars today is cold and dry. The transition to this present state is closely linked to the fate of the planet's surface water, which is poorly understood. A substantial amount of surface water escaped to space from the atmosphere, in part because of Mars' relatively low gravity ${ }^{1}$. However, atmospheric-escape models account for only some of ancient Mars' surface water. On page 391, Wade et al. ${ }^{2}$ propose that much of the surface water was sequestered underground.

Erupted lava had the chance to interact with surface water to form hydrated crust on both Earth and ancient watery Mars. Wade et al. examine the thermodynamic properties of the water-bearing 'mafic' crusts (which largely consist of the rock basalt) of each planet, and show that Martian basalt can hold more water than terrestrial basalt, and can effectively transport it to a greater depth below the surface (more than 90 kilometres; Fig. 1). The authors also compute the stability of water-containing minerals in hydrated crusts and their bulk-rock densities along both planets' geotherms (which describe how temperature varies with depth). They conclude that the burial of hydrated crusts progressively hydrates the interior of Mars, but that this process does not work effectively for Earth.
The four rocky planets of the Solar System (Mercury, Venus, Earth and Mars) are thought to have been formed by the accretion of similar planetary building blocks. This resulted in their mantles having broadly similar compositions of all the major elements, except for iron. Metallic iron partitions into the metallic cores of the rocky planets, whereas iron(II) oxide accumulates in silicate-rich planetary mantles. A thermodynamic property known as oxygen fugacity, which is a measure of the amount of oxygen present in a mixture, controls iron content in planetary basalts. Martian basalts have

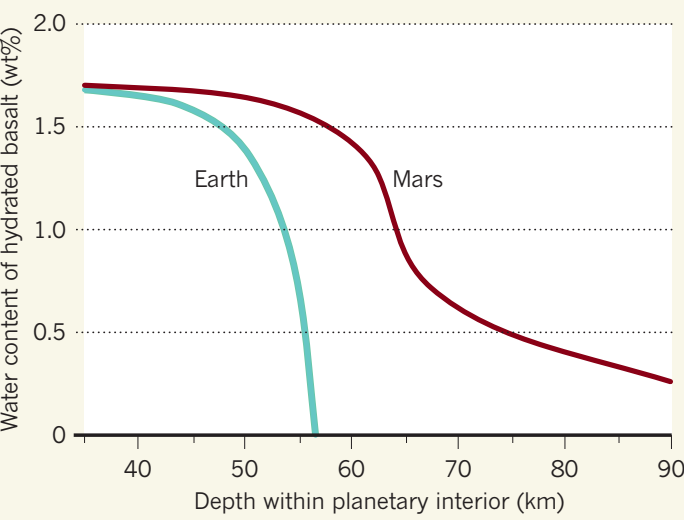

Figure 1 | The water content of basalt on Earth and Mars. Wade et al. ${ }^{2}$ report modelling of the surface evolution of Earth and Mars, which allowed them to estimate the amount of water that can be sequestered by basalt rock in the planets' interiors. They find that the Martian basalts can store more water (shown as percentage by weight), and at greater depths, than their terrestrial counterparts. This suggests that much of the water thought to have existed on the surface of ancient Mars was sequestered underground. (Adapted from Fig. 1a of ref. 2.) a distinctly higher content of iron(II) oxide (about $17 \%$ by weight) than do typical basalts on Earth (about $7-10 \%$ by weight), which suggests that oxygen fugacity was higher during Martian core formation than it was during Earth's core formation. Wade and colleagues show that this compositional difference, along with the different geotherms of Mars and Earth, has a key role in the storage and transportation of planetary surface water in the crust and mantle of the two planets.

Hydration processes generally expand the crustal volume, making the crust less dense. By contrast, Wade and colleagues' thermodynamic modelling indicates that the iron-rich Martian basalts undergo small volume expansions during hydration. Furthermore, hydrated iron-rich Martian basalts tend to melt at lower temperatures (about $800-900^{\circ} \mathrm{C}$ ) than does anhydrous mafic rock (about $1,200^{\circ} \mathrm{C}$ ), and this melting leaves relatively dense hydrated residues in the mantle. In the apparent absence on Mars of tectonic processes that recycle crust material into the mantle, the authors propose that successive burial of hydrated crust might have induced hydration of the mantle - such burial would have gradually increased the temperature and pressure applied to hydrated crustal basalts, causing them to melt, and therefore to leave hydrated residues in the mantle.

Hydration of the Martian mantle would also lead to it becoming more oxidized. However, geochemical analysis of meteorites (known as shergottites) formed from young Martian basalts suggest that their source in the mantle is less oxidized than Earth's mantle ${ }^{3}$. Moreover, the shergottite source is depleted in water (less than 50 parts per million $)^{4}$ relative to Earth's mantle (typically about $100-200$ p.p.m.). How do these observations fit into Wade and colleagues' suggestion that the Martian mantle contains more water than Earth's? Experiments have shown ${ }^{6}$ that the shergottite source is located at a depth of approximately $100 \mathrm{~km}$, which is near the base of the hydrated mantle column proposed by Wade and co-workers. The lower oxidation and hydration of the shergottite source might therefore be representative of (or place 